\title{
Oscilador Harmônico Duplo difuso
}

\author{
Diffuse Double-harmonic oscillator
}

\author{
Hercules Alves Oliveira ${ }^{* 10}$, Guilherme Jurkevicz Delben ${ }^{2}$ \\ ${ }^{1}$ Universidade Tecnolóogica Federal do Paraná, Ponta Grossa, PR, Brasil \\ ${ }^{2}$ Universidade Federal de Santa Catarina, Curitibanos, SC, Brasil
}

\begin{abstract}
Recebido em 04 de Julho de 2019. Revisado em 07 de Outubro de 2019. Aceito em 16 de Outubro de 2019.
A descrição do comportamento de um sistema difuso, linear e não-linear, pode ser tratada através da equação de Fokker-Planck. Esta equação tem despertado grande interesse de pesquisadores por sua boa adaptação a diferentes sistemas, tanto clássicos como quânticos. Neste trabalho, utilizamos a equação de Fokker-Planck para encontrar as probabilidades totais do potencial biestável difuso sob ação de um campo elétrico externo. As soluções do sistema mostram que existem duas funções de onda, uma simétrica e outra antissimétrica e quando o campo elétrico é aplicado todos os niveis de energia tornam-se degenerados. Observa-se que a aplicação do campo elétrico externo acarreta numa diminuição da temperatura do sistema e, consequentemente, um tempo menor para este atingir o equilíbrio.
\end{abstract}

Palavras-chave: Fokker-Planck, Potencial Biestável, Difusão.

The behavior description of diffuse system, linear and nonlinear, can be treated through Fokker-Planck equation. This equation has shown great interesting of researchers for its good adaptation to different systems, classical and quantum. In this work we use the Fokker-Planck to find the total probabilities of diffuse bistable potential under action of a external electric field. The system solutions show that there is two wave functions, one symmetrical and another anti-symmetrical and when the electric field is applied all energy levels are degenerate. It is observed that the application of external electric field entails in a decrease of temperature of the system and, consequently, shorter time to reach equilibrium.

Keywords: Fokker-Planck, Bistable Potential, Diffusion.

\section{Introdução}

Uma partícula confinada em um potencial harmônico duplo é um exemplo de um potencial biestável, tanto na física clássica quanto na física quântica. O potencial de confinamento é caracterizado por dois mínimos separados por uma barreira de energia de altura $V_{0}$. A informação mais importante de interesse em tal sistema é a dinâmica da distribuição populacional em ambos os mínimos. Classicamente, o único mecanismo que permite a passagem de um poço para o outro é o salto ativado termicamente sobre a barreira. Por outro lado, a característica peculiar do regime quântico é o tunelamento, o que permite que as amplitudes de probabilidade se espalhem através da barreira [1]. Recentemente, a dinâmica dos sistemas quânticos biestáveis voltou a ganhar interesse na literatura [2 3]. O interesse no potencial biestável é justificado por poder ser utilizado, qualitativamente, como um modelo físico simples, para simular o comportamento de diferentes sistemas mais complexos, entre eles a molécula de amônia (NH3) em um sistema denso [4], dissipação em sistemas quânticos abertos [5], além de sistemas de interesse para informação quântica, como o qubit [6] .

*Endereço de correspondência: hercules@utfpr.edu.br
Um sistema quântico que usa um potencial biestável já foi utilizado para entender o processo de quantum annealing [6], também para operação de portas quânticas, onde os dois primeiros estados do oscilador duplo, simétrico e antissimétrico, representam o sistema de dois níveis característico do qubit [7].

O uso de uma equação de Fokker-Planck caracterizada por uma distribuição de equilíbrio tem sido útil há décadas para modelar sistemas diferentes em mecânica estatística sem equilíbrio, mecânica quântica, astrofísica, ou até mesmo, áreas diversas, como química, biologia ou finanças e economia. Esta lista inclui, especificamente, reações químicas como isomerização, bistabilidade ótica, difusão no potencial biestável, caracterização de proteínas, e sistemas que podem exibir criticidade [8 10].

No presente trabalho pretendemos utilizar a equação de Fokker-Planck, na forma clássica, para encontrar as probabilidades totais do potencial biestável difuso, como proposto em [4], sob ação de um campo externo.

$\mathrm{Na}$ seção 2 apresentamos o potencial que define o oscilador harmônico duplo e as funções de onda que representam as soluções da equação de Schrödinger para o potencial biestável. Na seção 3 estuda-se a equação de Fokker-Planck e probabilidades totais do sistema quando este está submetido a um campo elétrico. Na seção 4 
apresentamos os resultados numéricos para o sistema com presença de campo elétrico. Na seção 5 apresentamos a conclusão do trabalho.

\section{O oscilador harmônico duplo}

O potencial do oscilador harmônico duplo é dado por:

$$
V(x)=\frac{1}{2} m \omega^{2}(|x|-\alpha)^{2} .
$$

O valor de $\alpha$ na Eq. (1) define a altura da barreira de potencial que separa os dois mínimos. Este parâmetro também define as posições em que ocorrem os mínimos do potencial.

Na Fig. 1 podemos visualizar o potencial para três valores distintos de $\alpha$, com $\alpha=\frac{4}{\sqrt{2}}, \frac{5}{\sqrt{2}}$ e $\frac{6}{\sqrt{2}}$ apresentados na forma de linha sólida, circulos e quadrados, respectivamente. Podemos visualizar que quanto maior o valor de $\alpha$ mais distante da barreira em $x=0$ está o ponto mínimo $(V(\alpha)=0)$. O potencial possui um valor bem definido para $x=0$ dado por $V(0)=V_{0}=\frac{1}{2} m \omega^{2} \alpha^{2}$. Que é o valor da barreira de potencial. Outra forte característica são os mínimos de potencial para $x=-\alpha$ e $x=\alpha$, onde o potencial é nulo. Podemos visualizar tais características na Fig. 2,

Para encontrar as autoenergias e autofunções relacionados ao potencial definido pela Eq. (1) iremos resolver a equação de Schrödinger independente do tempo, definida por [1]:

$$
-\frac{\hbar^{2}}{2 m} \frac{d^{2} \psi}{d x^{2}}+V(x) \psi(x)=E \psi(x) .
$$

Substituindo a Eq. (1) na Eq. (2), temos

$$
-\frac{\hbar^{2}}{2 m} \frac{d^{2} \psi}{d x^{2}}+\frac{1}{2} m \omega^{2}(|x|-a)^{2} \psi(x)=E \psi(x) .
$$

Este problema já foi resolvido e amplamente discutido na referência [11.

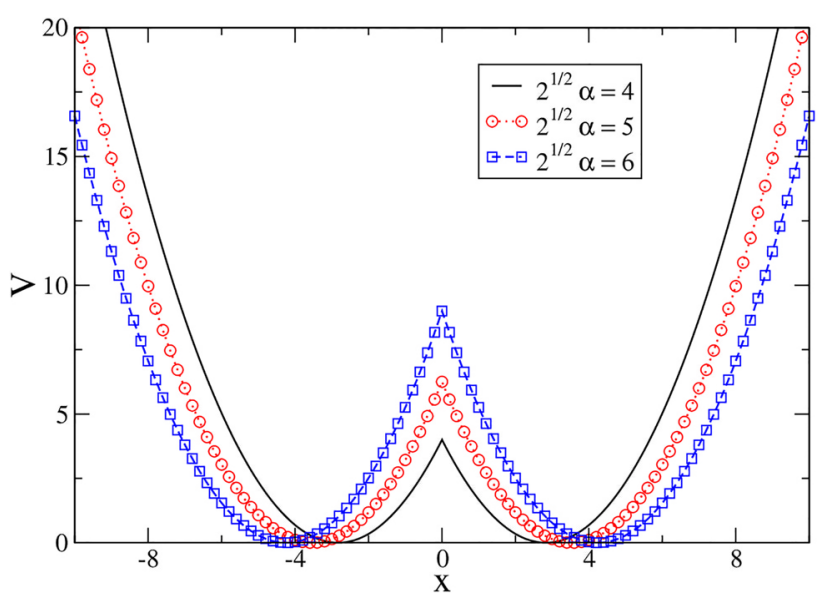

Figura 1: O potencial do oscilador harmônico duplo para diferentes tamanhos de barreira, $\alpha=\frac{4}{\sqrt{2}}, \frac{5}{\sqrt{2}}$ e $\frac{6}{\sqrt{2}}$.

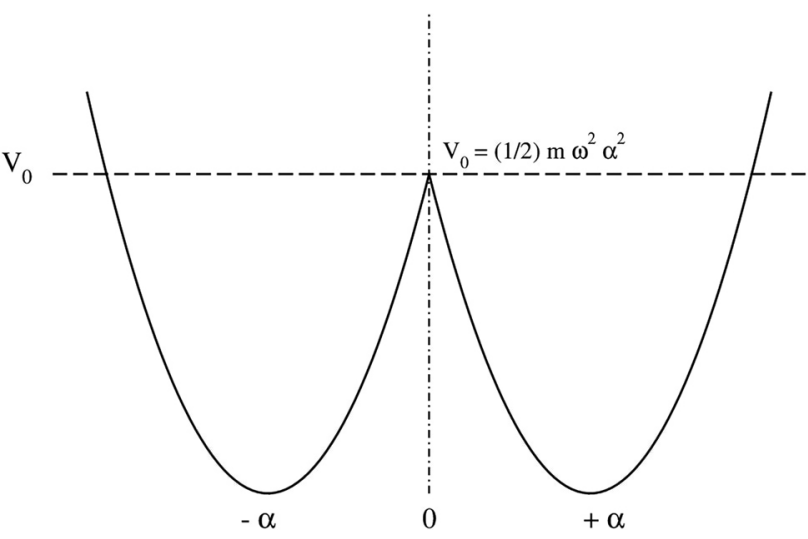

Figura 2: Representação do potencial apresentado na Eq. (1), onde podemos visualizar o potencial $V_{0}$ e os mínimos de potencial $V(-\alpha)=V(\alpha)=0$.

Os níveis de energia são dados por

$$
E=\hbar \omega\left(v_{n}+\frac{1}{2}\right)
$$

em termos do número quântico $v_{n}$ que, em geral, não é um número inteiro. Na Fig. 3 podemos visualizar os valores do número quântico $v_{n}$ em função do nível $n$ que se encontra para diferentes valores de barreira, $\alpha$. $\mathrm{Na}$ ampliação podemos visualizar que para os níveis de energia iniciais, $n$ pequeno, há uma duplicação dos valores de $v_{n}$. Essa degenerescência é causada pelo overlap das funções de onda do oscilador harmônico, à direita e à esquerda da barreira do potencial, que será apresentada a seguir.

Os autovalores de energia dados pela Eq. (4) tem a mesma forma dos encontrados para o caso do oscilador harmônico simples, de potencial $V(x)=\frac{1}{2} m \omega^{2} x^{2}$ e autoenergia $E_{n}=\hbar \omega\left(n+\frac{1}{2}\right)$, mas para pequenos valores de $n$ e $\alpha$ alguns autovalores são duplamente degenerados.

Quando $\alpha \rightarrow \infty$ todos os autovalores tornam-se duplamente degenerados [11]. Uma vez que o sistema pode

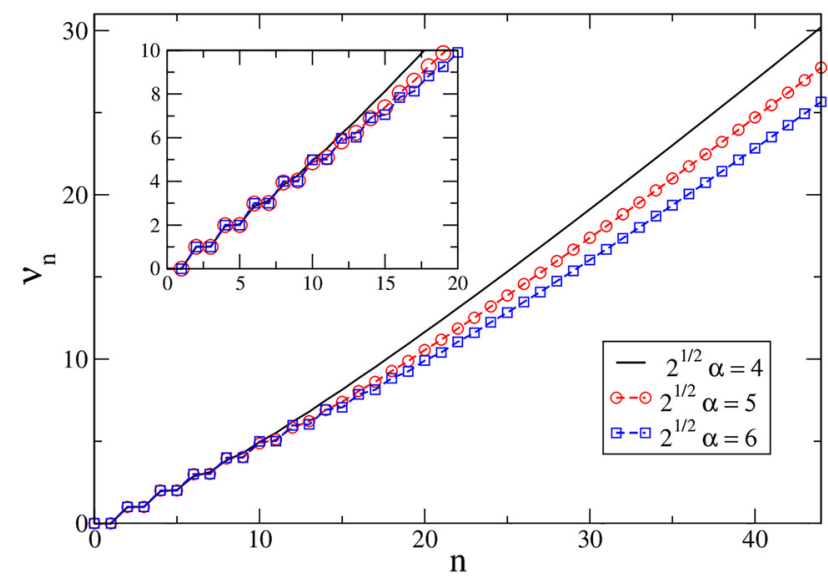

Figura 3: Valores do número quântico $v_{n}$ em função do nível de energia $n$ em que se encontra para diferentes valores de barreira $\alpha$. No quadro menor os valores de $n$ de 0 a 20 . 
ocupar um autoestado tanto no oscilador harmônico à esquerda quanto à direita. Tal comportamento já foi visto na Fig. 3, nos valores do número quântico $v_{n}$. Também pode-se visualizar a dupla degenerescência, para os menores autovalores, na Fig. 4, para $\alpha=\frac{4}{\sqrt{2}}$.

O potencial harmônico duplo é expresso por uma função par, portanto ele é invariante perante a reflexão dos valores de $x$. Entretanto, os autoestados são funções pares ou ímpares. A distribuição de probabilidade para cada autovalor de energia é simétrica com relação à origem, e nesses estados há igual probabilidade de encontrar o sistema em qualquer um dos dois poços de potencial. Se o estado tem maior probabilidade em um dos lados do potencial, o que temos é uma superposição de estados estacionários pares (simétricos) e ímpares (antissimétricos). A superposição é, em geral, não estacionária. Estas características do potencial viabilizam a caracterização física de um qubit.

Para resolver a equação de Schrödinger (Eq. (3p) iremos utilizar $\hbar=m=\omega=1$ e, já com estas considerações, definir a variável $z_{1}(x)$ para $x \geq 0$ e $z_{2}(x)$ para $x \leq 0$, assim

$$
\begin{aligned}
& z_{1}(x)=\sqrt{2}(x-\alpha), \text { se } x \geq 0, \\
& z_{2}(x)=\sqrt{2}(x+\alpha), \text { se } x \leq 0 .
\end{aligned}
$$

Com a definição das novas variáveis $z_{1}(x)$ e $z_{2}(x)$ e substituindo a Eq. (4) e Eq. (6) na Eq. (3), podemos reescrever a equação de Schrödinger na forma

$$
\frac{d^{2} \psi(x)}{d z_{1}^{2}(x)}+\left(v+\frac{1}{2}-\frac{z_{1}^{2}(x)}{4}\right) \psi(x)=0 .
$$

Obviamente teremos uma segunda equação de Schrödinger para $z_{2}(x)$, mas por ser similar a Eq. (7) iremos omitir e retornar apenas com seu resultado.

Uma solução particular da Eq. (7) é dada pela função parabólica cilíndrica

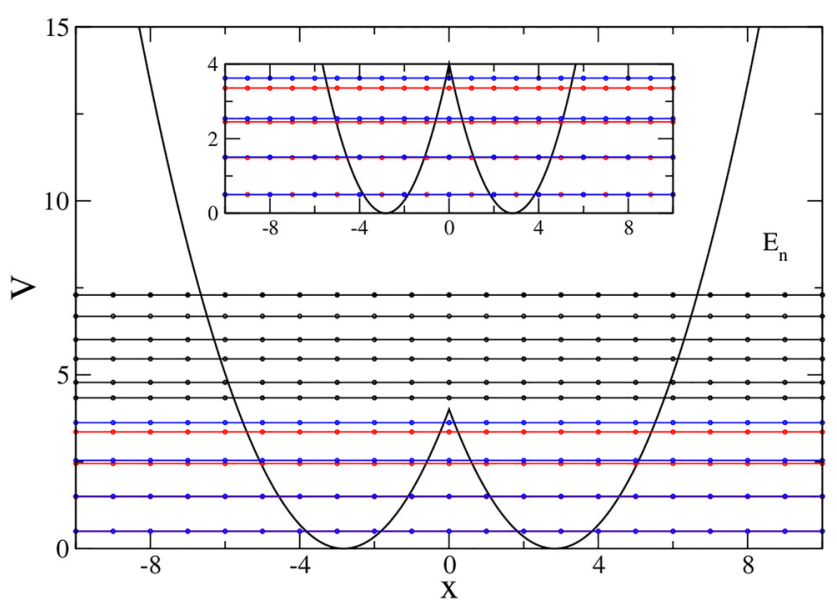

Figura 4: $O$ potencial e seus níveis iniciais de energia para $\alpha=\frac{4}{\sqrt{2}}$.

$$
\begin{aligned}
D_{v}\left(z_{1}\right) & =2^{\frac{v}{2}} e^{-\frac{z_{1}^{2}}{4}}\left[\frac{\Gamma\left(\frac{1}{2}\right)}{\Gamma\left(\frac{1-v}{2}\right)}{ }_{1} F_{1}\left(-\frac{v}{2} ; \frac{1}{2} ; \frac{z_{1}^{2}}{2}\right)+\right. \\
& \left.+\frac{z_{1}}{\sqrt{2}} \frac{\Gamma\left(-\frac{1}{2}\right)}{\Gamma\left(\frac{-v}{2}\right)}{ }_{1} F_{1}\left(-\frac{(1-v)}{2} ; \frac{3}{2} ; \frac{z_{1}^{2}}{2}\right)\right],
\end{aligned}
$$

onde ${ }_{1} F_{1}(a ; c ; z)$ é a função hipergeométrica confluente [12]. Durante os cálculos numéricos pode ser necessário expandir a função hipergeométrica confluente em séries, principalmente para o cálculo de integrais necessárias durante a normalização da função de onda. A expansão em série de ${ }_{1} F_{1}(a ; c ; z)$ é dada por 12

$$
{ }_{1} F_{1}(a ; c ; z)=1+\frac{a}{c} z+\frac{a(a+1)}{c(c+1)} \frac{z^{2}}{2}+\ldots,
$$

com $c \neq 0,-1,-2, \ldots$ Essa série é convergente para todo $z$ finito.

Desta forma, a solução da equação de Schrödinger será dada por

$$
\psi(x)=N_{n} \begin{cases}D_{v}(\sqrt{2}(x-\alpha)), & x \geq 0 \\ \pm D_{v}(-\sqrt{2}(x+\alpha)), & x \leq 0\end{cases}
$$

onde $N_{n}$ é a constante de normalização. Para $x \leq 0$ utilizamos o sinal positivo se, em $v_{n}, n$ par e o sinal negativo para $n$ ímpar.

Para encontrar a constante de normalização $N_{n}$ utilizamos a definição

$$
\int_{-\infty}^{\infty} \psi^{\dagger}(x, t) \psi(x, t) d x=1 .
$$

A Eq. (11) deve ser resolvida numericamente, utilizando a aproximação da função hipergeométrica confluente dada pela Eq. (9).

Os valores de $v_{n}$ e, consequentemente, os autovalores de energia são obtidos impondo a continuidade da função de onda e sua derivada em $x=0$. Este é o ponto onde os dois potenciais parabólicos tendem a ser descontínuos. Temos assim as seguintes condições:

$$
\begin{gathered}
\left.\frac{d D_{v}(\sqrt{2}(x-\alpha))}{d x}\right|_{x=0}=0 \text {, para } \psi(x) \text { par } \\
-D_{v}(-\sqrt{2} \alpha)=0 \text {, para } \psi(x) \text { ímpar }
\end{gathered}
$$

Os valores de $v_{n}$ apresentados na Fig. 3 foram obtidos por intermédio das Eq. 12 e Eq. 13.

As autofunções dos primeiros doze níveis de energia podem ser visualizadas na Fig. 5 Para tal, consideramos o caso onde a barreira de potencial é caracterizada por $\alpha=\frac{6}{\sqrt{2}}$, impondo ao problema uma barreira de potencial $V_{0}=18$. Este caso foi escolhido por fornecer uma boa 

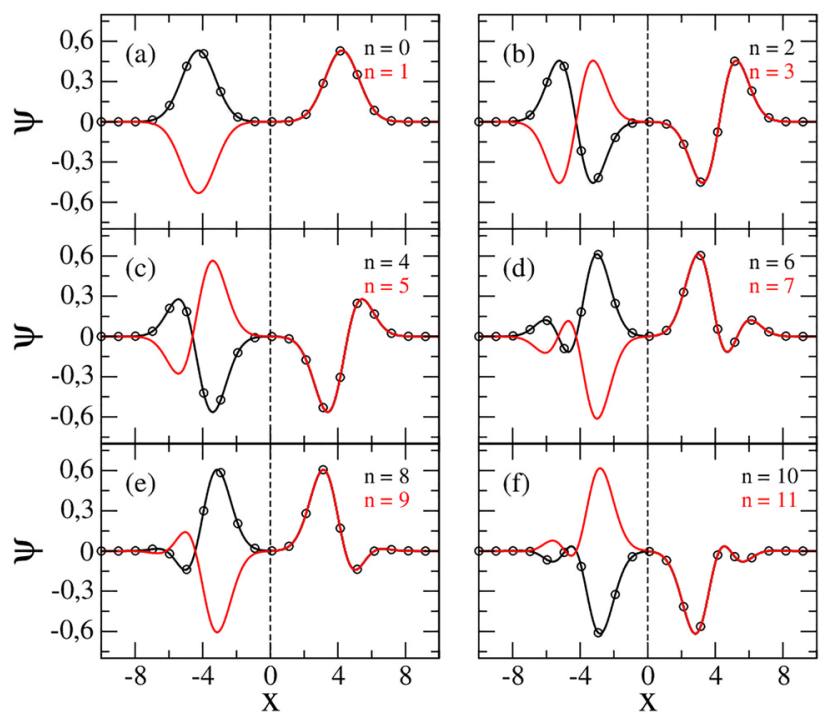

Figura 5: Autofunções para os primeiros doze níveis de energia para $\alpha=\frac{6}{\sqrt{2}}$.

caracterização dos autovalores de energia degenerados para $E_{n} \leq V_{0}$. Podemos visualizar na figura que para $x \leq 0$ a função de onda torna-se assimétrica entre os diferentes valores de $n$, pares ou ímpares.

Uma característica da equação de Schrödinger para potenciais simétricos com relação à origem é que as funções de onda têm simetrias bem definidas quanto aos lados negativo e positivo do potencial. As funções serão simétricas do lado direito do potencial e antissimétricas do lado esquerdo. Esta questão é bastante importante no cálculo das funções de onda pois, como podemos ver na Fig. 5 dois $n^{\prime} s$ consecutivos (veja Fig. 5 (a), $n=0$ e $n=1$ ) terão comportamentos idênticos do lado direito, mas opostos do lado esquerdo (antissimétricas).

Na Fig. 6 temos uma comparação entre as autofunções, deslocadas acima no potencial com valor proporcional ao seu nível de energia correspondente, para os primeiros níveis de energia entre (a) o oscilador harmônico duplo para $\alpha=\frac{6}{\sqrt{2}}$ e (b) o oscilador harmônico simples. Nesta figura podemos visualizar em $(a)$ que do lado direito do
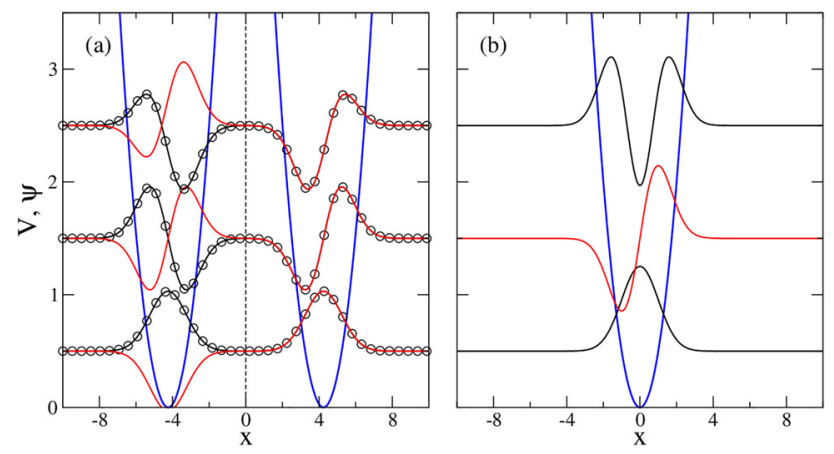

Figura 6: Comparação entre as autofunções para os primeiros níveis de energia entre (a) o oscilador harmônico duplo para $\alpha=\frac{6}{\sqrt{2}}$ e (b) o oscilador harmônico simples. potencial temos funções de onda simétricas, idênticas ao do oscilador harmônico simples $(b)$, enquanto do lado esquerdo $(a)$, temos funções de onda antissimétricas.

\section{Oscilador harmônico duplo sob ação do campo elétrico externo}

Considerando um oscilador harmônico duplo, unidimensional de carga $q$ e massa $m$, sujeito a ação de um campo elétrico externo em seu eixo de oscilação, $\vec{E}=E_{0} \hat{x}$.

Vamos considerar que o campo elétrico atuante sobre a partícula é constante, em cada intervalo de tempo $\delta t$, e igual a $E_{0}$. Dessa forma, a energia potencial $V_{E_{0}}(x)$ associada ao campo externo é

$$
V_{E_{0}}(x)=-q E_{0} x
$$

Assim, o potencial total que iremos substituir na equação de Schrödinger passa a ser dado por

$$
\begin{aligned}
V(x) & =V_{0}(x)+V_{E_{0}}(x) \\
& =\frac{1}{2} m \omega^{2}(|x|-\alpha)^{2}-q E_{0} x .
\end{aligned}
$$

Para $x>0$ temos

$$
V(x>0)=\frac{1}{2} m \omega^{2}\left(x^{2}-2 \alpha x+\alpha^{2}-\frac{2 q E_{0} x}{m \omega^{2}}\right)
$$

e para $x<0$ temos

$$
V(x<0)=\frac{1}{2} m \omega^{2}\left(x^{2}+2 \alpha x+\alpha^{2}-\frac{2 q E_{0} x}{m \omega^{2}}\right)
$$

o que resulta em

$$
V(x)=\frac{1}{2} m \omega^{2}\left[x^{2}+2 x\left(\mp \alpha-\frac{q E_{0}}{m \omega^{2}}\right)+\alpha^{2}\right],
$$

com o sinal $(-)$ para $x>0$ e $(+)$ para $x<0$. Adotaremos essa correspondência de sinais em todas as contas abaixo, sem adicionar um índice para não confundir o leitor.

O potencial proposto na Eq. (18) pode ser visualizado na Fig. 7. Este caso foi plotado considerando as seguintes constantes: $\hbar=m=\omega=q=1, \alpha=4 / \sqrt{2}$ e $E_{0}=0,1$.

Podemos escrever o potencial em função de um produto notável e, assim, tornar a Eq. 18 mais parecida com a Eq. (1), aproveitando a solução da equação de Schrödinger obtida anteriormente. 


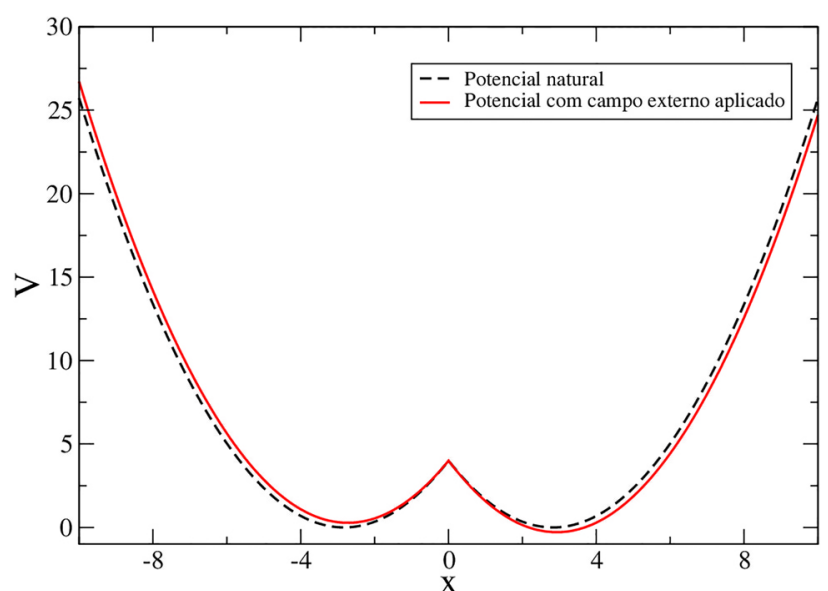

Figura 7: Potencial dado pela Eq. (18), com a atuação do campo externo.

$$
\begin{aligned}
V(x) & =\frac{m \omega^{2}}{2}\left\{\left[x+\left(\mp \alpha-\frac{q E_{0}}{m \omega^{2}}\right)\right]^{2} \mp \frac{2 \alpha q E_{0}}{m \omega^{2}}\right. \\
& \left.-\left(\frac{q E_{0}}{m \omega^{2}}\right)^{2}\right\} \\
& =\frac{m \omega^{2}}{2}\left[x+\left(\mp \alpha-\frac{q E_{0}}{m \omega^{2}}\right)\right]^{2} \mp \alpha q E_{0}-\frac{q^{2} E_{0}^{2}}{2 m \omega^{2}} \\
& =\frac{m \omega^{2}}{2}(x+\mathcal{A})^{2}+\mathcal{F},
\end{aligned}
$$

onde $\mathcal{A}$ e $\mathcal{F}$ são constantes, dadas por

$$
\mathcal{A}=\mp \alpha-\frac{q E_{0}}{m \omega^{2}}
$$

e

$$
\mathcal{F}=\mp \alpha q E_{0}-\frac{q^{2} E_{0}^{2}}{2 m \omega^{2}} .
$$

Nota-se que a Eq. (20) e a Eq. 21) podem ser aproximadas quando analisamos a ordem de grandeza de seus termos. Enquanto um valor médio que utilizamos para $\alpha$ é $4 / \sqrt{2}$, o maior valor de $E_{0}$ será 0,1 , e seu valor médio será bem menor. Então, podemos propor que $|\alpha| \gg-\frac{q E_{0}}{m \omega^{2}}$, assim, temos que $\mathcal{A}=\mp \alpha-\frac{q E_{0}}{m \omega^{2}} \approx \mp \alpha$. Da mesma forma, temos que $\mathcal{F} \approx \mp \alpha q E_{0}$. Lembrando que utilizamos o sinal $(-)$ para $x>0$ e $(+)$ para $x<0$.

Na Fig. 8 são apresentados os níveis de energia para o sistema quando um campo elétrico externo é aplicado, $\operatorname{com} \alpha=\frac{4}{\sqrt{2}}$ e $E=0,1$.

\subsection{Reescrevendo a equação de Schrödinger}

Com a aplicação do campo elétrico a equação de Schrödinger deve ser reescrita como

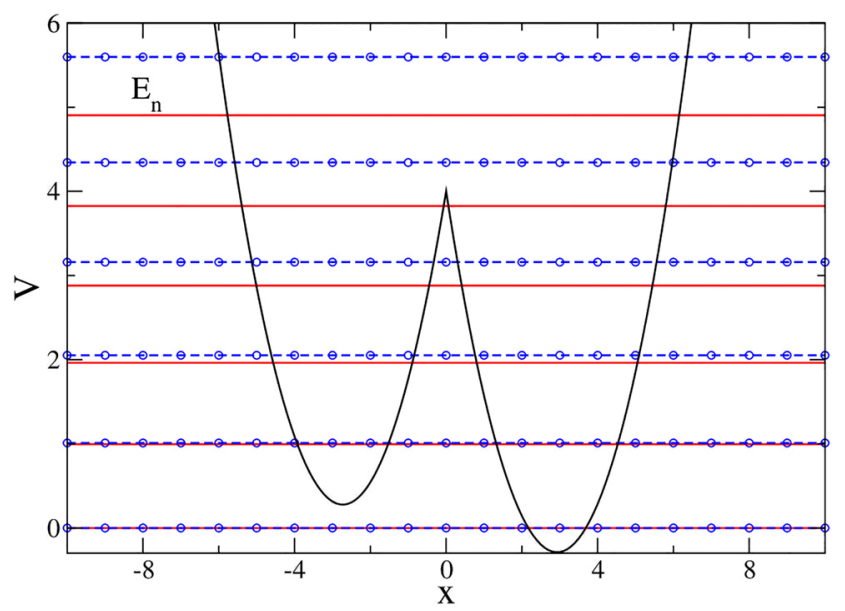

Figura 8: $O$ potencial e seus níveis iniciais de energia para $\alpha=\frac{4}{\sqrt{2}}$, com aplicação do campo esterno $E=0,1$.

$-\frac{\hbar^{2}}{2 m} \frac{\partial^{2} \psi(x)}{\partial x^{2}}+\frac{1}{2} m \omega^{2}(x+\mathcal{A})^{2} \psi(x)=\left(\lambda_{E_{0}}-\mathcal{F}\right) \psi(x)$,

onde $\lambda_{E_{0}}$ são os autovalores de energia do sistema com atuação do campo externo.

Comparando a Eq. (22) com a Eq. (3), temos uma equação de energia dada em função da Eq. (4), $\lambda_{n}=$ $\hbar \omega\left(v_{n}+1 / 2\right)$, que pode ser escrita por

$$
\lambda_{E_{0}}-\mathcal{F}=\lambda_{n},
$$

isolando a energia do sistema com a atuação do campo externo, temos

$$
\begin{aligned}
\lambda_{E_{0}} & =\lambda_{n}+\mathcal{F} \\
& =\hbar \omega\left(v_{n}+\frac{1}{2}\right) \mp \alpha q E_{0}-\frac{q^{2} E_{0}^{2}}{2 m \omega^{2}} .
\end{aligned}
$$

Quando $E_{0}=0$ temos que $\lambda_{E_{0}}=\lambda_{n}$, obviamente.

Desta forma, a solução da equação de Schrödinger, assim como na Eq. 10$)$, será dada por

$$
\psi(x)=N\left\{\begin{array}{ll}
D_{v}(\sqrt{2}(x+\mathcal{A})), & x \geq 0 \\
\pm D_{v}(-\sqrt{2}(x+\mathcal{A})), & x \leq 0
\end{array},\right.
$$

onde $N$ é a constante de normalização. A Eq. 25) é a solução do oscilador harmônico duplo que resolvemos anteriormente. Podemos ver que o campo elétrico constante aplicado no eixo de oscilação não altera a natureza harmônica do movimento, alterando apenas a energia e ponto de equilíbrio do sistema.

Utilizando $(\mathcal{A})$, podemos reescrever a Eq. 25 exatamente como a Eq. (10), quando o campo elétrico é nulo. O que será de grande valia durante a implementação numérica do problema, pois nos permitirá utilizar a Eq. (8), a Eq. (11), a Eq. (12) e a Eq. (13) sem dependência do campo externo. 


\subsection{A equação de Fokker - Planck}

A dinâmica do sistema fora do equilíbrio, como do oscilador harmônico duplo com difusão, pode ser estudada através da equação de Fokker-Planck. Em particular, a equação de Fokker-Planck é usada para tratar processos que envolvem difusão ou transferência de população.

A equação de Fokker-Planck é obtida através da equação de Langevin [13] e fornece a evolução temporal da distribuição de probabilidade do sistema de interesse. Devido à dificuldade de solucionar essa equação, podemos mapeá-la em uma equação do tipo Schrödinger. A associação da equação de Fokker-Planck com a equação de Schrödinger tem sido bastante útil não somente para potenciais com solução analítica, mas também para potenciais mais complicados, pela possibilidade do uso dos métodos de soluções aproximativos, por exemplo, o uso do formalismo de supersimetria e o método variacional [14].

Com a relação entre a equação de Fokker-Planck e a equação de Schrödinger, sistemas estudados na mecânica quântica fornecem subsídios para a análise de processos estocásticos. A vantagem desta associação é que se pode obter a solução da equação de Fokker-Planck por meio de métodos de solução da equação de Schrödinger, como proposto em [4].

A equação de Schrödinger associada à equação de Fokker-Planck a ser resolvida é dada por

$$
-\frac{\Gamma_{D}}{2} \frac{d^{2} \psi(x)}{d x^{2}}+V_{e f}(x) \psi(x)=-\Lambda_{n} \psi(x),
$$

sendo $\Gamma_{D}=2 D$, em que $D$ é o coeficiente de difusão. O termo referente ao potencial efetivo é igual ao potencial estudado $V_{E_{0}}(x)$, Eq. (19), já considerando que $\mathcal{A}=\alpha$, ou seja,

$$
V_{e f}(x)=\frac{1}{2}\left\{\frac{[f(x)]^{2}}{\Gamma_{D}}-\frac{\partial f(x)}{\partial x}\right\}=\frac{1}{2}(|x|-\alpha)^{2}+\mathcal{C} .
$$

Como mostrado na expressão acima, o potencial efetivo está relacionado a uma força $f(x)$, portanto, pode-se procurar uma força que forneça um potencial efetivo similar ao potencial original. A força sugerida neste caso tem a forma:

$$
f(x)=\beta+\gamma x,
$$

sendo que $\beta$ e $\gamma$ são parâmetros determinados pela seguinte equação,

$$
\frac{1}{2}\left\{\frac{(\beta+\gamma x)^{2}}{\Gamma_{D}}-\gamma\right\}=\frac{1}{2}(|x|-\alpha)^{2}+\mathcal{C}
$$

Resolvendo a Eq. 29 e comparando os termos, podemos escrever $f(x)$ como

$$
f(x)=-\sqrt{\Gamma_{D}}\left\{\begin{array}{ll}
(x-\alpha), & x>0 \\
(x+\alpha), & x<0
\end{array} .\right.
$$

Então, o potencial efetivo é dado por

$$
V_{e f}(x)=\frac{1}{2}\left\{(|x|-\alpha)^{2}-\sqrt{\Gamma_{D}}\right\} .
$$

Substituindo a Eq. (31) na Eq. (26), temos

$-\frac{\Gamma_{D}}{2} \frac{d^{2} \psi(x)}{d x^{2}}+\frac{1}{2}\left\{(|x|-\alpha)^{2}-\sqrt{\Gamma_{D}}\right\} \psi(x)=-\Lambda_{n} \psi(x)$ (32)

Considerando a equação de Schrödinger resolvida na seção anterior, Eq. (3), e adotando a seguinte troca de variáveis

$$
z(x)= \begin{cases}\sqrt{\frac{2}{\Gamma_{D}}}(x-\alpha), & x \geq 0 \\ \sqrt{\frac{2}{\Gamma_{D}}}(x+\alpha), & x \leq 0\end{cases}
$$

temos

$$
\frac{d^{2} \psi}{d z^{2}}+\left(\frac{\sqrt{\Gamma_{D}}}{2}-\Lambda_{n}-\frac{\Gamma_{D} z^{2}}{4}\right) \psi=0
$$

Comparando a Eq. (34) com a Eq. (7), precisamos efetuar as seguintes mudanças de variáveis

$$
\epsilon=-\left(\Lambda_{n}+\frac{1}{2}-\frac{\sqrt{\Gamma_{D}}}{2}\right)
$$

e

$$
\xi^{2}=\Gamma_{D} z^{2}= \begin{cases}\sqrt{2}(x-\alpha), & x \geq 0 \\ \sqrt{2}(x+\alpha), & x \leq 0 .\end{cases}
$$

Desta forma, podemos reescrever a Eq. (34) como

$$
\frac{d^{2} \psi}{d z^{2}}+\left(\epsilon+\frac{1}{2}-\frac{\xi^{2}}{4}\right) \psi=0
$$

Consequentemente, podemos escrever a solução particular da equação diferencial como uma função parabólica cilíndrica, dada por

$$
\begin{aligned}
D_{\epsilon}(\xi) & =2^{\frac{\epsilon}{2}} e^{-\frac{\xi^{2}}{4}}\left[\frac{\Gamma\left(\frac{1}{2}\right)}{\Gamma\left(\frac{1-\epsilon}{2}\right)}{ }_{1} F_{1}\left(-\frac{\epsilon}{2} ; \frac{1}{2} ; \frac{\xi^{2}}{2}\right)+\right. \\
& \left.+\frac{\xi}{\sqrt{2}} \frac{\Gamma\left(-\frac{1}{2}\right)}{\Gamma\left(\frac{-\epsilon}{2}\right)}{ }_{1} F_{1}\left(-\frac{(1-\epsilon)}{2} ; \frac{3}{2} ; \frac{\xi^{2}}{2}\right)\right] .
\end{aligned}
$$

Desta forma, a solução da equação de Fokker-Planck será dada por

$$
\psi(x)=N_{n} \begin{cases}D_{\epsilon}(\sqrt{2}(x-\alpha)), & x \geq 0 \\ \pm D_{\epsilon}(-\sqrt{2}(x+\alpha)), & x \leq 0\end{cases}
$$

onde $N_{n}$ é a constante de normalização. Para $x \leq 0$ utilizamos o sinal positivo se, em $\epsilon_{n}, n$ par e o sinal negativo para $n$ ímpar. 
Os autovalores de energia $\Lambda_{n}$ são obtidos da comparação direta da Eq. (37) com a Eq. (24), escrevendo que

$$
\frac{\sqrt{\Gamma_{D}}}{2 \Gamma_{D}}-\Lambda_{n}=\lambda_{E_{0}}+\epsilon_{0}
$$

Acrescentamos um termo constante $\epsilon_{0}$ ao potencial com o objetivo de deslocar os níveis de energia e obter o primeiro autovalor da equação como sendo nulo - desconsiderando o valor do campo elétrico. Esta operação não altera os resultados obtidos, apenas impõe uma condição que auxilia na descrição do estado estacionário.

Abrindo e simplificando a Eq. (40) podemos escrever os autovalores de energia do problema como

$$
\Lambda_{n}=-\left(v_{n}+\epsilon_{0}+\frac{1}{2}-\frac{\sqrt{\Gamma_{D}}}{2}+\alpha E_{0}-\frac{E_{0}^{2}}{2}\right)
$$

onde

$$
\epsilon_{0}=-\frac{1}{2}-v_{0}
$$

Finalmente, substituindo a Eq. (42) na Eq. (41), obtemos que os autovalores de energia são dados por

$$
\Lambda_{n}=v_{n}-v_{0}-\frac{\Gamma_{D}}{2}+\alpha E_{0}-\frac{E_{0}^{2}}{2},
$$

onde temos que os níveis de energia do sistema também dependem do campo externo e do coeficiente de difusão.

\subsection{Probabilidades Totais}

A equação de Fokker-Planck pode ser escrita como a seguinte equação diferencial

$$
\frac{\partial P(x, t)}{\partial t}=-\frac{\partial}{\partial x}[f(x) P(x, t)]+\frac{\Gamma_{D}}{2} \frac{\partial^{2} P(x, t)}{\partial x^{2}},
$$

onde $P(x, t)$ é a distribuição de probabilidade que descreve a dinâmica do potencial a ser estudado.

A solução geral da equação de Fokker-Planck [13] dependente do tempo pode ser escrita em termos da solução da equação de Schrödinger associada por

$$
P(x, t)=\psi_{0}(x) \sum_{n=0}^{\infty} b_{n} \psi_{n}(x) \exp \left[-\frac{t \Lambda_{n}}{\hbar}\right] .
$$

Para obter os coeficientes $b_{n}$ da somatória iremos multiplicar ambos os lados da Eq. (45) por $\psi_{l}(x) / \psi_{0}(x)$ e integrar com relação a $x$ considerando-se $t=0$,

$\int_{-\infty}^{\infty} P(x, 0) \frac{\psi_{l}(x)}{\psi_{0}(x)} d x=\int_{-\infty}^{\infty} \sum_{n=0}^{\infty} b_{n} \psi_{0}(x) \psi_{n}(x) \frac{\psi_{l}(x)}{\psi_{0}(x)} d x$

$$
\int_{-\infty}^{\infty} P(x, 0) \frac{\psi_{l}(x)}{\psi_{0}(x)} d x=\sum_{n=0}^{\infty} b_{n} \int_{-\infty}^{\infty} \psi_{n}(x) \psi_{l}(x) d x .
$$

Pela propriedade de ortogonalidade, a integral do lado direito é igual a um quando $n=l$ e igual a zero quando $n \neq 1$, ou seja,

$$
\int_{-\infty}^{\infty} \psi_{n}(x) \psi_{l}(x) d x=\delta_{n, l}
$$

Portanto,

$$
b_{n}=\int_{-\infty}^{\infty} P(x, 0) \frac{\psi_{n}(x)}{\psi_{l}(x)} d x .
$$

Neste ponto iremos escolher a distribuição inicial de probabilidade $P(x, 0)$, quando $t=0$. A equação de Fokker-Planck é satisfeira impondo como condição inicial a função delta de Dirac. Isto posto, vamos considerar a condição inicial

$$
P(x, 0)=\delta\left(x-x_{0}\right) .
$$

Sendo assim,

$$
b_{n}=\int_{-\infty}^{\infty} \delta\left(x-x_{0}\right) \frac{\psi_{n}(x)}{\psi_{0}(x)} d x .
$$

Finalmente, a equação final para os coeficientes $b_{n}$ é dada por

$$
b_{n}=\frac{\psi_{n}\left(x_{0}\right)}{\psi_{0}\left(x_{0}\right)} .
$$

Note que, para um potencial menos complexo, como o oscilador harmônico simples, poderíamos utilizar uma versão simplificada da Eq. 45, não dependente de $\psi_{0}(x)$, que forneceria uma equação para os coeficientes $b_{n}$ independente da simetria das funções de onda, implícita nos termos em função de $x_{0}$ na divisão $\frac{\psi_{n}\left(x_{0}\right)}{\psi_{0}\left(x_{0}\right)}$. Neste caso, o termo $\psi_{0}\left(x_{0}\right)$ irá indicar em qual lado do potencial está a função de onda inicial e, consequentemente, a simetria da mesma. Logo após os instantes iniciais o pacote irá se dividir entre os dois lados do potencial até atingir o equilíbrio.

Substituindo a Eq. 52 na Eq. 45, obtemos

$$
P(x, t)=\sum_{n=0}^{\infty} \psi_{n}\left(x_{0}\right) \psi_{n}(x) \exp \left[-\frac{t \Lambda_{n}}{\hbar}\right] .
$$

Com o objetivo de observar a evolução temporal do sistema iremos calcular as probabilidades totais em função do tempo através das integrais do lado esquerdo $(x<0)$ e do lado direito $(x>0)$ da distribuição de probabilidade, sendo $N_{1}(t)$ e $N_{2}(t)$, respectivamente. Portanto, temos as seguintes equações

$$
N_{1}(t)=\int_{-x_{L}}^{0} P(x, t) d x,
$$




$$
N_{2}(t)=\int_{0}^{x_{L}} P(x, t) d x
$$

onde $x_{L}$ é o limite de integração.

Podemos definir a diferença de probabilidade do sistema como

$$
\delta N(t)=N_{1}(t)-N_{2}(t),
$$

concluimos que o sistema atingirá um estado estacionário quando $\delta N(t)=0$. O tempo para atingir o equilíbrio define a condição de que metade da amostra está do lado esquerdo $(x<0)$ e a outra metade está do lado direito $(x>0)$ do potencial. Esse resultado é esperado, uma vez que se trata de um potencial biestável simétrico em que as probabilidades são iguais nos mínimos do potencial.

As probabilidades totais $N_{1}(t)$ e $N_{2}(t)$ são importantes pois demonstram claramente o comportamento natural do sistema de dividir a população e encontrar o equilíbrio entre os dois lados do potencial. Também que, quando o equilíbrio é atingido, a amostra recai em um estado estacionário $(\delta N(t)=0)$.

\section{Resultados e discussão}

Para as simulações numéricas iremos adotar que $\hbar=$ $m=\omega=q=1$. Uma das principais medidas a serem estudadas será a diferença de probabilidade $\delta N(t)$, definida na Eq. (56). Utilizando o intervalo de integração $x_{L}=10$, que define a região onde temos a maior barreira de potencial no sistema (ver Fig. 7). Enquanto a posição inicial do pacote será, em todos os casos estudados, $x_{0}=-\alpha$. Iremos considerar que quando $\delta N(t) \leq 0,001$ o sistema atingiu o estado estacionário e o tempo onde a desigualdade é satisfeita será chamado de $t_{s s}$.

\subsection{Efeito do campo externo na distribuição de probabilidade}

Nesta seção iremos adotar que $\Gamma_{D}=1$. Isto posto, considerando o efeito do campo externo na distribuição de probabilidade, temos a diferença de probabilidade $\delta N(t)$ apresentada na Fig. 9 Para diferentes valores de campo externo quando a barreira de potencial é $\alpha=\frac{4}{\sqrt{2}}$. Em $(a)$, $E_{0}=0$, é o caso sem aplicação de campo externo, resultado conhecido e apresentado em [4]. Em (b), $E_{0}=0,001$, reduzindo a metade o tempo que o sistema leva para atingir o estado estacionário. No último caso apresentado, (c), temos o maior valor utilizado para o campo elétrico externo neste trabalho, $E_{0}=0,1$. Neste caso, o tempo necessário para o sistema atingir o estado estacionário é cerca de 400 vezes menor que o caso sem campo externo. Isto mostra o possibilidade de controle do sistema através do campo externo.

A Fig. 10 é complementar aos resultados expostos acima. Nesta figura temos o valor do tempo necessário para o sistema atingir o estado estacionário, $t_{s s}$, relacionado ao valor do campo elétrico aplicado. Nova-
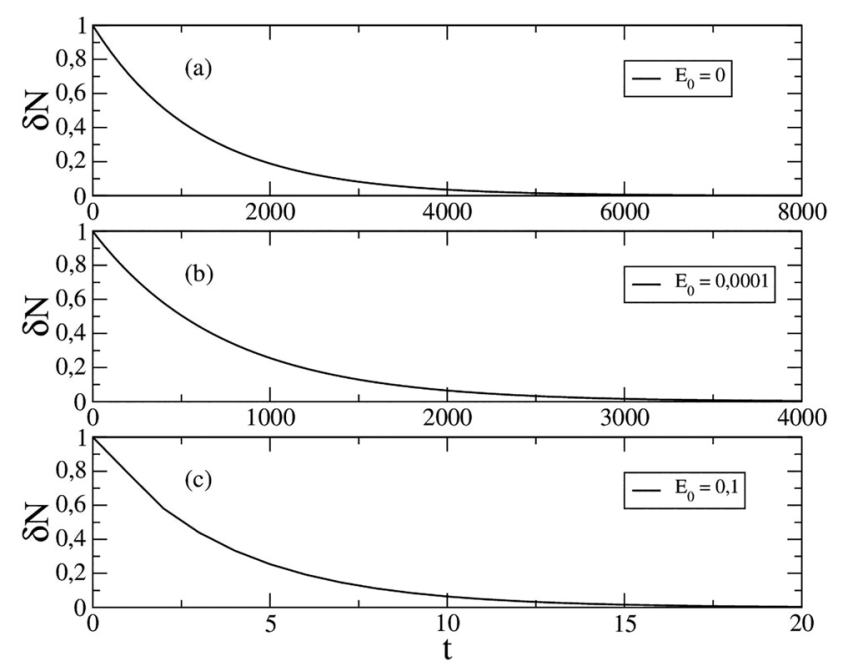

Figura 9: Diferença de probabilidade $\delta N$ para alguns valores de campo externo quando a barreira de potencial é $\alpha=\frac{4}{\sqrt{2}}:$ (a) $E_{0}=0$, (b) $E_{0}=0.0001$, (c) $E_{0}=0.1$.

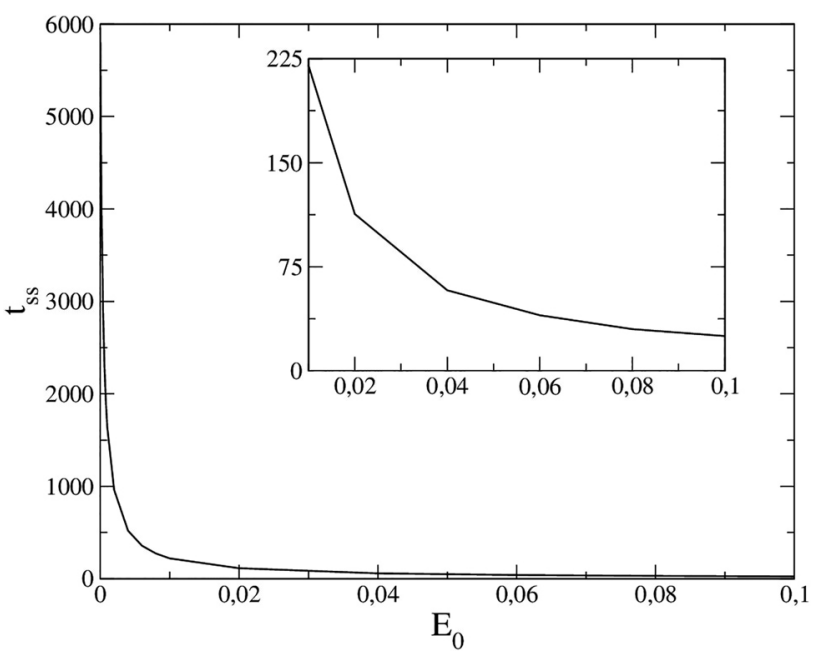

Figura 10: Tempo necessário para o sistema atingir o estado estacionário, $t_{s s}$, dependendo do valor de campo externo aplicado.

mente quando a barreira de potencial utilizada é $\alpha=\frac{4}{\sqrt{2}}$. Quanto maior o campo aplicado menor o tempo necessário para o sistema atingir o estado estacionário.

\subsection{Variando a difusão do sistema}

Para estimar o tempo que o sistema leva para atingir o estado estacionário em diferentes regimes de difusão iremos evoluir o sistema para diferentes valores do coeficiente de difusão, $0 \leq \Gamma_{D} \leq 4$. A evolução temporal é considerada até que $\delta N(t) \leq 0,001$, que é quando o sistema atinge o estado estacionário. Como anteriormente, chamaremos o tempo em que o estado estacionário é alcançado de $t_{s s}$.

Na Fig. 11 vemos, em $(a)$, o comportamento de $t_{s s}$ contra $\Gamma_{D}$. Concluímos que quanto maior o coeficiente de difusão maior o tempo necessário para que o sistema atinja o estado estacionário. Para compreender este resul- 


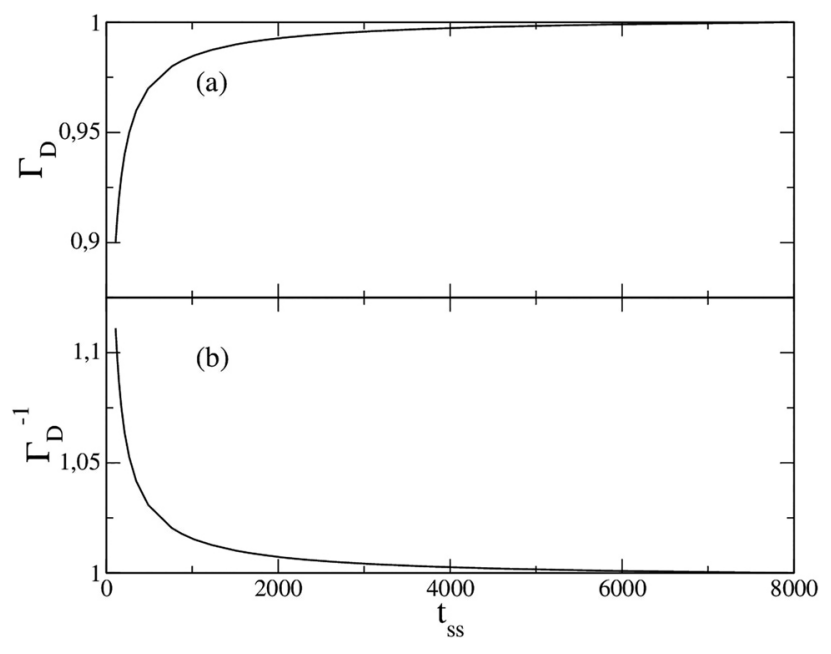

Figura 11: (a) Tempo necessário para o sistema atingir o estado estacionário contra o valor da difusão. (b) Tempo necessário para o sistema atingir o estado estacionário contra o inverso da difusão, que é proporcional à temperatura do sistema.

tado é importante termos em mente a função da difusão no espalhamento do pacote de onda. Quando o coeficiente de difusão é menor ocorrerá pouco espalhamento do pacote de onda e o sistema levará um tempo menor para atingir o equilíbrio. Quando o coeficiente de difusão é maior o pacote de onda se espalha mais dentro do potencial e leva mais tempo para que ele atinja o equilíbrio. Observa-se que uma coisa é observar o tempo de difusão, outra é o tempo que o pacote de onda leva para atingir o equilíbrio.

Na Fig. 11 (b) utilizamos uma escala proporcional ao inverso da temperatura, $\Gamma_{D}^{-1}$, o que mostra que quanto maior a temperatura do sistema maior o tempo necessário para o sistema atingir o estado estacionário.

Observa-se que quanto maior o coeficiente de difusão, maior será o tempo necessário para o sistema atingir o estado estacionário. Contudo, quanto maior o campo elétrico, menor será a difusão do sistema. Obviamente estamos tentando associar o campo externo com as propriedades físicas inerentes ao sistema. Com isso, podemos dizer que a aplicação do campo externo diminui o tempo necessário para o sistema atingir o estado estacionário, tornando-o equivalente a um sistema com menor regime de difusão, consequentemente com menor temperatura média.

\section{Conclusão}

Neste trabalho apresentamos o comportamento do Oscilador Harmônico Duplo com a aplicação de um campo elétrico externo. As equações de probabilidade do sistema foram modeladas com a equação de Fokker-Planck sob efeito de um campo externo. É conhecido na literatura que, para este tipo de sistema, quando o tempo que sistema leva para atingir o estado estacionário é longo, relaxamento lento, apenas as primeiras autofunções do sistema são relevantes. Enquanto, quando o tempo que o sistema leva para atingir o estado estacionário é curto, relaxamento rápido, as autofunções de maior ordem possuem grande influência no comportamento das probabilidades totais do sistema 15. Este comportamento ficou bem evidenciado em nossos resultados. A aplicação do campo externo torna as autofunções de maior ordem no sistema mais relevantes, levantando os níveis de energia do sistema e alterando o tempo de relaxamento.

O efeito apresentado pela aplicação do campo externo apresentou resultados interessantes para o tempo de relaxamento do sistema, que é o tempo necessário para o sistema atingir o estado estacionário. $\mathrm{O}$ resultado mais importante aqui exposto é o de que o campo externo é permitido apenas para reduzir o tempo natural de relaxamento do sistema. Qualquer tentativa de aplicação para que aumente o tempo necessário para o equilíbrio das populações do sistema não é permitido.

Outro fenômeno discutido é a equivalência matemática, no tempo necessário para que $\delta N(t) \approx 0$, entre a aplicação do campo externo e o coeficiente de difusão do sistema. Podemos dizer que o efeito produzido pelo campo externo no sistema é equivalente a reduzir a temperatura média do sistema. Estes fenômenos podem ser indicativos dos efeitos do controle quântico em sistemas abertos [16].

Este trabalho baseia-se, obviamente, em um modelo físico bastante simples. Entretanto, os efeitos aqui discutidos podem servir, qualitativamente, para pensarmos nos problemas existentes em sistemas físicos mais complexos. Este pode ser um modelo para estudo de limitações do controle quântico em sistemas quânticos dissipativos. Como, por exemplo, a perda de controle em um qubit, o controle de difusão em modelos do tipo Caldeira Leggett, ou sistemas baseados no modelo de gato de Schrödinger 17,22 .

\section{Apêndice A: Modificando a configuração do campo externo}

Consideramos que o campo elétrico atuante sobre a partícula é constante, em cada intervalo de tempo $\delta t$, e igual a $E_{0}$. Estudamos para a configuração do campo elétrico externo em que $V_{E_{0}}(x) \propto x$. Entretanto, podemos discutir outras configurações para o campo externo. Neste apêndice iremos apresentar outras duas configurações e discutir as diferenças e similaridades com o caso estudado no trabalho.

Com tal intuito iremos considerar agora dois casos, nos quais $V_{E_{1}}(x) \propto|x|$ e $V_{E_{2}}(x) \propto(|x|-\alpha)$. Na Fig. 12 temos uma comparação entre o potencial do oscilador harmônico duplo, Eq. (1), e os três casos discutidos do potencial sob ação de um campo elétrico externo, $V_{E_{0}}$ (Eq. (14)), $V_{E_{1}}$ (Eq. (57)), e $V_{E_{2}}$ (Eq. (61)). Estes casos foram plotados considerando as seguintes constantes: $\hbar=\omega=q=1, \alpha=4 / \sqrt{2}$ e $E_{0}=0,1$. 


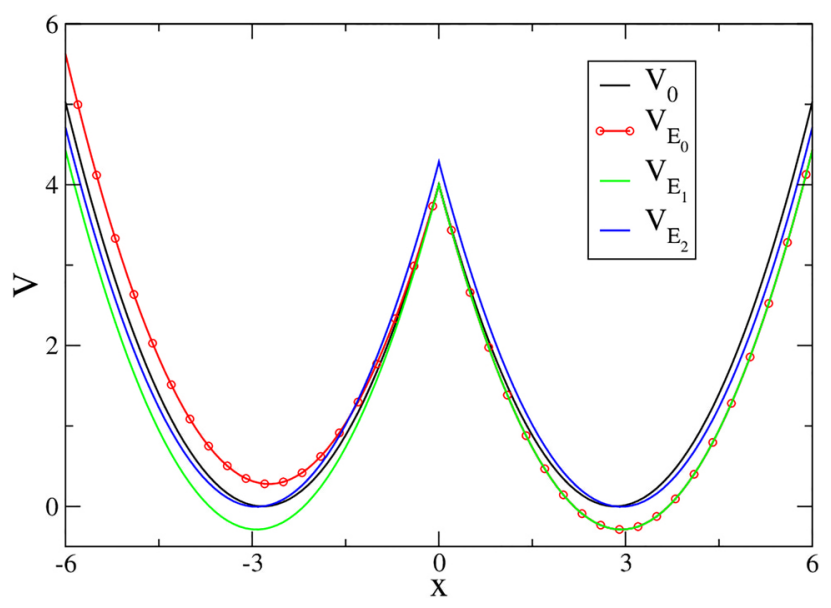

Figura 12: Comparação entre o potencial do oscilador harmônico duplo, Eq. (1), e os três casos discutidos do potencial sob ação de um campo elétrico externo, $V_{E_{0}}$ (Eq. (14)), $V_{E_{1}}$ (Eq. (57)), e $V_{E_{2}}$ (Eq. (61)).

Dessa forma, o potencial $V_{E_{1}}(x)$ associado ao campo externo é

$$
V_{E_{1}}(x)=-q E_{0}|x| .
$$

Utilizando da mesma lógica utilizada para o potencial da Eq. 14 e definindo $c=\frac{m \omega^{2}}{2}$, teremos

$$
\begin{aligned}
V(x) & =c\left\{\left[|x|-\left(\alpha+\frac{q E_{0}}{m \omega^{2}}\right)\right]^{2}-\frac{2 \alpha q E_{0}}{m \omega^{2}}-\left(\frac{q E_{0}}{m \omega^{2}}\right)^{2}\right\} \\
& =c\left[|x|-\left(\alpha+\frac{q E_{0}}{m \omega^{2}}\right)\right]^{2}-\alpha q E_{0}-\frac{q^{2} E_{0}^{2}}{2 m \omega^{2}} \\
& =c\left(|x|-\mathcal{A}_{1}\right)^{2}+\mathcal{F}_{1},
\end{aligned}
$$

onde $\mathcal{A}_{1}$ e $\mathcal{F}_{1}$ são constantes, dadas por

$$
\mathcal{A}_{1}=\alpha+\frac{q E_{0}}{m \omega^{2}}
$$

e

$$
\mathcal{F}_{1}=-\alpha q E_{0}-\frac{q^{2} E_{0}^{2}}{2 m \omega^{2}}
$$

Para que tenhamos a mesma configuração do potencial da Eq. (19): $\left(x+\mathcal{A}_{1}\right)^{2}, \mathcal{A}_{1}$ deve ser escrito como $\mathcal{A}_{1}=\mp\left(\alpha+\frac{q E_{0}}{m \omega^{2}}\right)$, com o sinal $(-)$ para $x>0$ e $(+)$ para $x<0$, com $\mathcal{F}_{1}$ sendo o mesmo.

Uma terceira configuração de campo elétrico pode ser definida por

$$
V_{E_{2}}(x)=-q E_{0}(|x|-\alpha) .
$$

Utilizando da mesma lógica utilizada para o potencial da Eq. (14), teremos

$$
\begin{aligned}
V(x) & =c\left\{\left[|x|-\left(\alpha+\frac{q E_{0}}{m \omega^{2}}\right)\right]^{2}-\left(\frac{q E_{0}}{m \omega^{2}}\right)^{2}\right\} \\
& =c\left[|x|-\left(\alpha+\frac{q E_{0}}{m \omega^{2}}\right)\right]^{2}-\frac{q^{2} E_{0}^{2}}{2 m \omega^{2}} \\
& =c\left(|x|-\mathcal{A}_{2}\right)^{2}+\mathcal{F}_{2},
\end{aligned}
$$

onde $\mathcal{A}_{2}$ e $\mathcal{F}_{2}$ são constantes, dadas por

$$
\mathcal{A}_{2}=\alpha+\frac{q E_{0}}{m \omega^{2}}=\mathcal{A}_{1},
$$

e

$$
\mathcal{F}_{2}=-\frac{q^{2} E_{0}^{2}}{2 m \omega^{2}}
$$

Mesmo variando os sinais da $x$, para o lado positivo e para o lado negativo, $\mathcal{A}_{2}=\mathcal{A}_{1}$. Este mesmo comportamento não é visto para $\mathcal{F}_{2}$ devido às diferentes simetrias dos potenciais. Note que no potencial com $x$ sem módulo (Eq. (20)), a mudança de lado está presente no sinal de $\alpha$ e nos potenciais com módulo o sinal é aplicado a todo o termo de $\mathcal{A}_{1,2}$ (Eq. 59 e Eq. (63)).

Comparando ao resultado apresentado anteriormente no trabalho, observa-se que o termo $\mathcal{A}$ é semelhante ou igual, dependendo do potencial adotado para o campo elétrico Eq. (20), Eq. (59) e Eq. (63). Desta forma, podemos comparar os resultados para o termo $\mathcal{F}$ em Eq. (21), Eq. (60) e Eq. (64). Os termos $\mathcal{F}$ e $\mathcal{F}_{1}$ são semelhantes a menos de um sinal em $\alpha$, estes dependem da largura da barreira $(\alpha)$. Consequentemente, não deve alterar o comportamento ou ordem de grandeza do campo externo necessário para alterar o tempo de equilíbrio do sistema. Já o termo $\mathcal{F}_{2}$, Eq. (64), não depende da largura da barreira de potencial, com isto, tem uma influência muito menor no sistema, sendo necessário maiores intensidades do campo elétrico.

\section{Apêndice B: Oscilador Harmônico quântico}

O oscilador harmônico simples é um dos sistemas mais estudados em física tanto no tratamento clássico quanto no quântico. Este sistema é frequentemente abordado em livros de Mecânica Quântica por ser possível obter sua solução analítica e por ser utilizado em aproximações de potenciais mais complexos como em sistemas que apresentam vibrações de moléculas ou estruturas nucleares 11].

O potencial do oscilador harmônico simples é dado por:

$$
V(x)=\frac{1}{2} m \omega^{2} x^{2}
$$

onde $m$ é a massa da partícula sob influência do potencial e $\omega$ é a frequência angular. A equação de Schrödinger 
independente do tempo, para uma partícula num estado $|\psi(x)\rangle$ no espaço de Hilbert, é dada por [23]:

$$
-\frac{\hbar^{2}}{2 m} \frac{d^{2} \psi}{d x^{2}}+\frac{1}{2} m \omega^{2} x^{2} \psi(x)=E \psi(x),
$$

onde a função de onda da partícula é definida como $\psi(x)=\langle x \mid \psi(x)\rangle$ e $E$ é a energia do sistema.

A solução do sistema é obtida definindo $b=(\hbar / m \omega)^{1 / 2}$, $\eta=\frac{m E b^{2}}{\hbar^{2}}=\frac{E}{\hbar \omega}$ e fazendo a mudança de variáveis $x=b y$, obtendo

$$
\frac{d^{2} \psi}{d y^{2}}+\left(2 \eta-y^{2}\right) \psi=0
$$

Após algumas análises da equação diferencial e das funções resultantes, que não trataremos aqui, mas que podem ser consultadas nas referências [11 23 24], a solução da Eq. (67), na variável original, é dada por

$$
\psi_{n}(x)=\left(\frac{m \omega}{2^{2 n}(n !)^{2} \hbar \pi}\right)^{1 / 4} H_{n}\left(\sqrt{\frac{m \omega}{\hbar}} x\right) e^{\left(-m \omega x^{2} / 2 \hbar\right)},
$$

onde $H_{n}$ são os polinômios de Hermite [12,24].

A energia do sistema é dada por

$$
E_{n}=\left(n+\frac{1}{2}\right) \hbar \omega
$$

A representação gráfica das funções de onda do oscilador harmônico pode ser verificada na Fig. 13 para os níveis (a) $n=0$, (b) $n=1$, (c) $n=2$, (d) $n=3$, (e) $n=4$ e em (f) $n=5$.
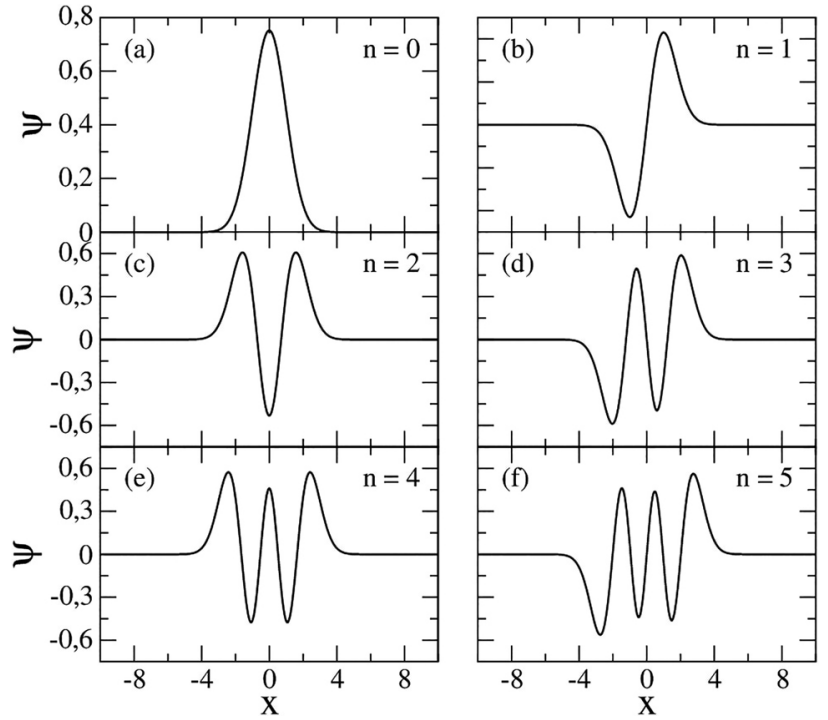

Figura 13: Funções de onda do oscilador harmônicos simples para os níveis de $n=0$ a $n=5$.

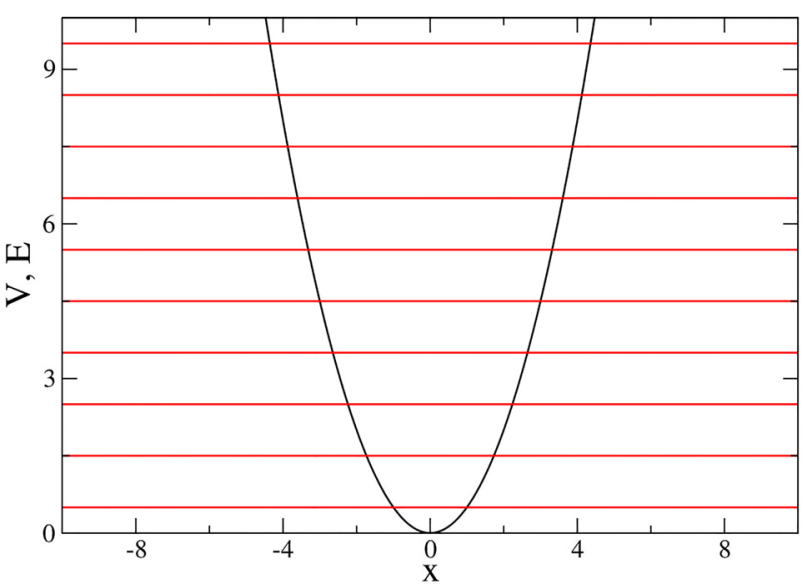

Figura 14: O potencial do oscilador harmônico simples e os níveis de energia.

As funções de onda apresentam uma característica bem específica para sua paridade, quando $n$ for par, as funções serão pares, ou seja, $\psi(-x)=\psi(x)$. E para $n$ ímpar, as funções serão ímpares $\psi(-x)=-\psi(x)$.

Para $n=0$, o polinômio de Hermite é dado por $H_{0}=1$, fazendo com que a função de onda seja proporcional a uma exponencial de $-x^{2}$. Para $n$ maior, $H_{n>1}$ é proporcional a polinômios de $x$, de diferentes ordens, como em $n=1$, onde $H_{1}$ é proporcional a $x$. Esses polinômios fornecem a oscilação da função de onda dentro do limite do potencial.

Os níveis de energia em comparação com o potencial são vistos na Fig. 14. Um ponto interessante desse sistema é que os níveis de energia são discretos e estão igualmente espaçados, com $E=\frac{\hbar \omega}{2}, \frac{3 \hbar \omega}{2}, \frac{5 \hbar \omega}{2}, \cdots$. Outro ponto é que o nível de menor energia, chamado de estado fundamental para o menor estado ligado da partícula com o potencial, com $n=0, E=\frac{\hbar \omega}{2}$, não coincide com o mínimo do potencial.

\section{Referências}

[1] L. Magazzu, D. Valenti, A. Carollo e B. Spagnolo, Entropy 17, 02341 (2015).

[2] S. Bera, S. Florens, H.U. Baranger, N. Roch, A. Nazir e A.W. Chin, Phys. Rev. B 89, 121108 (2014).

[3] A.H. Hosseinloo e K. Turitsyn, Smart Mater. Struct. 25, 015010 (2016).

[4] D. Caldas, J. Chahine e E. Drigo Filho, Physica A 412, 92 (2014).

[5] D. Kast e J. Ankerhold, Phys. Rev. Lett. 110, 010402 (2010).

[6] W.D. Oliver, Nature 473, 164 (2011).

[7] J. Mompart, K. Eckert, W. Ertmer, G. Birkl e M. Lewenstein, Phys. Rev. Letters 90, 147901 (2003).

[8] M.T. Araujo e E. Drigo Filho, Condensed Matter Physics 18, 43003 (2015).

[9] B.D. Shizgal, J. Stat. Phys. 164, 1379 (2016).

[10] F. Polotto, E. Drigo Filho, J. Chahine e R.J. de Oliveira, Physica A 483, 286 (2018). 
[11] E. Merzbacher, Quantum Mechanics (John Wiley and Sons, New York, 1997), $3^{\text {a }}$ ed.

[12] G.B. Arfken e H.J. Weber, Física Matemática (Elsevier, Rio de Janeiro, 2007), $6^{\mathrm{a}}$ ed.

[13] T. Tomé e M.J. Oliveira, Dinâmica Estocástica e Irreversibilidade (EdUsp, São Paulo, 2014), $2^{\text {a }}$ ed.

[14] S.R.A. Salinas, Introdução à Mecânica Estatística (EdUsp, São Paulo, 2013).

[15] B.U. Felderhof, Physica A 387, 5010 (2008).

[16] H.A. Oliveira e G.J. Delben, Chinese J. Phys. 60, 141 (2019).

[17] C.A. Estrada Guerra, D. Velasco Villamizar e L.G.C. Rego, Phys. Rev. A 86, 023411 (2012).

[18] S.J. Glaser, U. Boscain, T. Calarco, C.P. Koch, W. Köckenberger, R. Kosloff, I. Kuprov, B. Luy, S. Schirmer, T. Schulte-Herbrüggen et al., Eur. Phys. J. D. 69, 379 (2015).

[19] F. Gottwald, S.D. Ivanov e O. Kuhn, J. Phys. Chem. Lett. 6, 2722 (2015).

[20] P. Massignan, A. Lampo, J. Wehr e M. Lewenstein, Phys. Rev. A 91, 033627 (2015).

[21] F. Minganti, N. Bartolo, J. Lolli, W. Casteels e C. Ciuti, Sci. Reports 6, 26987 (2016)

[22] I. Vega e D. Alonso, Rev. Mod. Phys. 89, 015001 (2017).

[23] C. Cohen-Tannoudji, B. Diu e F. Laloë Quantum Mechanics (John Wiley and Sons, Nova York, 2005), v. 1.

[24] R. Shankar, Principles of Quantum Mechanics (Plenum Press, New York, 1994), 2rd ed. 